\title{
Cobalt-related impurity centers in diamond: electronic properties and hyperfine parameters
}

\author{
R. Larico ${ }^{(1)}$, L. V. C. Assali(1), W. V. M. Machado(1) ${ }^{(1)}$ and J. F. Justo ${ }^{(2)}$ \\ (1) Instituto de Física, Universidade de São Paulo, \\ CP 66318, CEP 05315-970, São Paulo, SP, Brazil \\ (2) Escola Politécnica, Universidade de São Paulo, \\ CP 61548, CEP 05424-970, São Paulo, SP, Brazil
}

\begin{abstract}
Cobalt-related impurity centers in diamond have been studied using first principles calculations. We computed the symmetry, formation and transition energies, and hyperfine parameters of cobalt impurities in isolated configurations and in complexes involving vacancies and nitrogen atoms. We found that the Co impurity in a divacant site is energetically favorable and segregates nitrogen atoms in its neighborhood. Our results were discussed in the context of the recently observed Co-related electrically active centers in synthetic diamond.

PACS numbers: 61.72.Lk, 61.72.Nn, 61.72.Bb, 62.20.Fe
\end{abstract}




\section{INTRODUCTION}

Semiconducting materials featuring wide bandgaps have potential technological applications, such as ultraviolet radiation sources [1] or high-voltage switching devices [2]. Among those materials, diamond has also received considerable attention from a fundamental standpoint, due to a combination of superior physical properties. It has the largest known bulk modulus, high thermal conductivity and chemical inertness. Growing diamond out of graphite has been achieved by the high pressure-high temperature (HPHT) methods. In order to speed up the process and allow to get macroscopic samples, 3d-transition metal alloys have been used as solvent-catalysts. Those transition metals (TM) end up contaminating the samples, generating electrically and optically active centers [3]. Nickel was the first transition metal impurity unambiguously identified in synthetic diamond [4], ever since, several nickel-related active centers have been observed by electron paramagnetic resonance (EPR) [5-7] and optical [8] measurements. Although cobalt has been the most widely used solvent-catalyst to grow diamond [9], cobalt-related defects could not be identified as easily as the nickel-related ones. It was usually considered that either cobalt atoms were incorporated in very low concentrations to allow a proper detection or that the cobalt-related centers were electrically and/or optically inactive. However, recent investigations have identified several cobalt-related active centers in synthetic diamond [10-13].

The presence of cobalt impurities in diamond was first reported in the literature by EPR measurements [14]. The unique feature of the hyperfine structure was associated to a nuclear spin $\mathrm{I}=7 / 2$, a trace of ${ }^{59} \mathrm{Co}$ nuclei, and the center would be formed by an isolated interstitial cobalt in an octahedral distorted environment, in a doubly positive charged state $\left(\mathrm{Co}_{\mathrm{i}}^{2+}\right)$ with a $3 \mathrm{~d}^{7}$ configuration. More recently, a cobalt-related center, labeled O4 [11], was identified in HPHT diamond using EPR measurements. The proposed microscopic model for this center, with a spin $\mathrm{S}=1 / 2$ in a monoclinic $\left(\mathrm{C}_{1 \mathrm{~h}}\right)$ symmetry, is a cobalt atom in a semi-divacancy site $\left(\mathrm{C}_{3} V \mathrm{Co} V \mathrm{C}_{3}\right)$ plus a nitrogen atom replacing one of the Co six nearest neighboring carbon atoms, in the negative charge state $\left(\mathrm{C}_{3} V \operatorname{Co} V \mathrm{C}_{2} \mathrm{~N}\right)^{-}[13]$. Although there were no resolved nitrogen-related EPR lines, the nitrogen was proposed to pertain to this center due to two reasons: the presence of nitrogen atoms in the complexes could explain the symmetry lowering of the $\left(\mathrm{C}_{3} V \mathrm{Co} V \mathrm{C}_{3}\right)$ precursor complex and the resulting center would be similar to those previously identified NE centers, that were related to nickel-nitrogen complexes 
[15]. Two additional cobalt-related active centers have been identified in diamond, labeled as NLO2 and NWO1 [13]. The similarity in the EPR spectra of O4, NLO2, and NWO1, all of them with spin $\mathrm{S}=1 / 2$, indicates that all those centers should involve a cobalt atom in a semi-divacancy site with different number of neighboring nitrogen atoms. Therefore, the microscopic model for either NLO2 and NWO1 was suggested as $\left(\mathrm{NC}_{2} V \mathrm{Co} V \mathrm{C}_{2} \mathrm{~N}\right)^{0}$ or $\left(\mathrm{C}_{3} V \mathrm{Co} V \mathrm{CN}_{2}\right)^{0}$, with $\mathrm{C}_{2 \mathrm{~h}}$ or $\mathrm{C}_{1 \mathrm{~h}}$ symmetry, respectively. Table $\llbracket$ summarizes the available experimental data on the Co-related electrically active centers in diamond.

In order to understand the nature of those cobalt-related centers, and eventually control their activity, it is important to have a deep knowledge on their configurations, how they interact with other defects and/or impurities, and the respective microscopic processes of formation. Theoretical models can provide important information on those issues, such as the role of those defects in the electronic and optical properties of the material and how nitrogen atoms interact with those centers. Here, we used first principles total energy calculations to investigate the properties of several cobalt-related centers. Our results on symmetry, spin, formation and transition energies, and hyperfine parameters were compared to the properties of active centers recently identified in diamond.

\section{METHODOLOGY}

Earlier theoretical investigations on transition metals in diamond have been focused on nickel-related defect centers [16-19], only very recently cobalt-related centers have received some attention [20 22]. Here, we carried an investigation on the cobalt-related centers using the all-electron spin-polarized full-potential linearized augmented plane wave (FP-LAPW) method, implemented in the WIEN package [23]. The calculations were performed within the framework of the density functional theory and the generalized gradient approximation, using the Perdew-Burke-Ernzerhof exchange-correlation potential [24], and includes spin-orbit coupling in a second-variational procedure. All the calculations were performed considering a 54-atom reference supercell. We used atomic spheres for all atoms with radius of $\mathrm{R}=1.2$ a.u., a value that allowed simulations with large relaxations and distortions without any atomic sphere overlap. We additionally considered a $2 \times 2 \times 2 \mathrm{k}$-point grid to sample the irreducible Brillouin zone, as well as the $\Gamma$-point.

Convergence on the total energy was achieved using an augmented-plane-wave basis to 
describe the interstitial region, with the number of plane-waves limited by the 7.0/R parameter. Self-consistent interactions were performed until total energy and total charge in the atomic spheres changed by less than $10^{-4} \mathrm{Ry} /$ atom and $10^{-5}$ electronic charges/atom between two iterations, respectively. The atomic positions were relaxed until the forces were smaller than $10^{-3}$ Ry/a.u., with no symmetry constrains. Those approximations and convergence criteria have provided an accurate description of several defect centers in semiconductors [25 27]. Formation and transition energies of all centers were computed using the procedure discussed in ref. [25]. This procedure requires the total energies of the respective defect center and the chemical potentials of carbon, nitrogen and cobalt. Those chemical potentials were computed using the total energy of carbon in a diamond lattice, nitrogen in a $\mathrm{N}_{2}$ molecule, and cobalt in a hcp lattice, all computed within the FP-LAPW methodology with those same convergence criteria.

The isotropic hyperfine fields $\left(A_{i s o}\right)$ were computed as a combination of the contact term plus the isotropic part of the orbital term, since that for transition metals the orbital contribution is not quenched and cannot be neglected.

\section{ELECTRONIC AND STRUCTURAL PROPERTIES}

\section{A. Isolated Cobalt}

We start considering the cases of an isolated substitutional cobalt impurity. According to symmetry considerations, in a tetrahedral crystalline environment, the 3d-related Co atomic orbitals split into a three-fold $t_{2}$ plus a two-fold $e$ irreducible representations. Since Co interacts strongly with the nearest neighboring carbon atoms, the $e$ states are located below the $t_{2}$ ones. While the $e$ levels remain non-bonding, the d-related $t_{2}$ states interact with the bandgap vacancy-related $t_{2}$ ones, that came from the dangling bonds on the carbon host atoms surrounding the vacant site into which the impurity was introduced. The resulting structure comprises of a bonding $t_{2}$ level, resonant in the valence band (VB), plus an antibonding vacancy-like $t_{2}$ level in the bandgap. This electronic structure is similar to that of a substitutional nickel impurity in diamond [18], both being well described by the vacancy model [28].

In the positive charge state $\left(\mathrm{Co}_{\mathrm{s}}^{+}\right)$, the center is stable in $\mathrm{T}_{\mathrm{d}}$ symmetry and presents a 
closed shell configuration $(\mathrm{S}=0)$. There are three cobalt-related energy levels: the nonbonding $e$ and the bonding $t_{2}$ levels are resonant in the $\mathrm{VB}$, while the anti-bonding $t_{2}$ one remains unoccupied in the bandgap [22]. In the neutral charge state, the center presents spin $\mathrm{S}=1 / 2$ in a near tetragonal symmetry, with the unpaired electron occupying the antibonding $t_{2 \uparrow}$. In the negative charge state, the center presents a spin $\mathrm{S}=1$ in a near tetragonal symmetry. In the doubly negative charge state $\left(\mathrm{Co}_{\mathrm{s}}^{2-}\right)$, the center has a $\mathrm{T}_{\mathrm{d}}$ symmetry and a spin $\mathrm{S}=3 / 2$. Table $\llbracket$ summarizes all those results.

The electronic structure of the $\mathrm{Co}_{\mathrm{s}}^{2-}$ center is similar to that of $\mathrm{Ni}_{\mathrm{s}}^{-}$(known as the W8 center in the literature) [5, 18], having a $t_{2 \uparrow}^{3} t_{2 \downarrow}^{0}$ bandgap configuration. In that sense, the $\mathrm{Co}_{\mathrm{s}}^{2-}$ center should be identified by EPR measurements as easily as the Ni-related W8 center has been [5], but no EPR signal has been associated to such a Co-related center so far. This apparent paradox could be explained by analyzing the respective positions of the transition energies with relation to the nitrogen donor transition, that lies at $\varepsilon_{\mathrm{v}}+3.8 \mathrm{eV}$, where $\varepsilon_{\mathrm{v}}$ is the VB top. The $\mathrm{Ni}_{\mathrm{s}}(0 /-)$ transition is at $\varepsilon_{\mathrm{v}}+3.0 \mathrm{eV}$, therefore below the nitrogen donor level, but the $\mathrm{Co}_{\mathrm{s}}(-/ 2-)$ one is at $\varepsilon_{\mathrm{v}}+4.0 \mathrm{eV}$, above that donor level. As a result, the doubly negative charge state of $\mathrm{Co}_{\mathrm{s}}$ is probably unaccessible. The electronic structure of the $\mathrm{Co}_{\mathrm{s}}^{-}$ center is similar to that of $\mathrm{Ni}_{\mathrm{S}}^{0}$ [18], both with spin $\mathrm{S}=1$, but there is no evidence, from EPR measurements, from either one. The symmetry lowering-related splitting between the two highest occupied levels in $\mathrm{Co}_{\mathrm{s}}^{-}$and $\mathrm{Ni}_{\mathrm{s}}^{0}$ is very small and may explain why those centers have not been observed so far, as discussed in Refs. [29, 30]. The $\mathrm{Co}_{\mathrm{s}}^{0}$, with a spin $\mathrm{S}=$ $1 / 2$, could be experimentally observed by boron codoping, in order to provide compensated samples.

According to table III, the formation energies of isolated cobalt in interstitial sites $\left(\mathrm{Co}_{\mathrm{i}}\right)$ are considerably larger than those in substitutional ones. This result is typical for transition metal impurities in semiconductors with small lattice parameters, such as diamond [18], silicon carbide [25], and boron nitride [27]. Although this would suggest a prevailing concentration of substitutional TMs, growth conditions of HPHT diamond could still lead to interstitial TM impurities.

The 3d-related electronic levels of $\mathrm{Co}_{\mathrm{i}}$ centers present a $t_{2}$ state below the $e$ one. This indicates that the interstitial cobalt interacts more weakly with its nearest neighbors than with its next nearest neighbors, suggesting that the Ludwig-Woodbury (LW) model is well suited to describe these centers [31]. On the other hand, in all charge states, the 3d-related 
levels occupations do not follow the Hund's rule and $\mathrm{Co}_{\mathrm{i}}$ presents a low spin (LS) ground state configuration, which follows from the prevailing crystal field splitting over the exchange one. These results contrast with those of TM impurities in silicon, in which both the LW model and the Hund's rule are generally followed.

For all $\mathrm{Co}_{\mathrm{i}}$ charge states, the $t_{2}$ levels are fully occupied, being resonant in the VB. In the negative charge state, $\mathrm{Co}_{\mathrm{i}}^{-}$presents $\mathrm{T}_{\mathrm{d}}$ symmetry, being diamagnetic $(\mathrm{S}=0)$. In the neutral charge state, $\mathrm{Co}_{\mathrm{i}}^{0}$ presents a spin $\mathrm{S}=1 / 2$ and tetragonal symmetry. In the positive charge state, $\mathrm{Co}_{i}^{+}$presents a $\mathrm{T}_{\mathrm{d}}$ symmetry and a spin $\mathrm{S}=1$, with the highest occupied energy level being an $e_{\uparrow}^{2}$ near the VB top. In the doubly positive charge state, $\mathrm{Co}_{\mathrm{i}}^{2+}$ presents a spin $\mathrm{S}=3 / 2$ and tetragonal symmetry. For the latest case, the Co impurity behaves as an acceptor impurity, leaving a polarized hole in the valence band top. This behavior could be explored in the context of high temperature ferromagnetism mediated by free carriers in semiconductors [32].

Several Co-related active centers appear in HPHT diamond after thermal annealing at $\mathrm{T} \approx 1800^{\circ} \mathrm{C}$. It has been suggested that the resulting centers are aggregates of Co impurities and vacancies, that became mobile under such conditions [13]. Annealing under even higher temperatures $\left(\mathrm{T} \approx 2000^{\circ} \mathrm{C}\right)$ leads to diffusion of nitrogen impurities, which pair with those Co-vacancy complexes. Figure 1a presents the atomic configuration of a Co impurity between two carbon vacancies, which is labeled as $\left(\mathrm{C}_{3} V \mathrm{Co} V \mathrm{C}_{3}\right)$. Atoms from 1 to 6 represent the six neighboring carbon atoms of the Co impurity. They could be better described as two sets of carbons in a trigonal symmetry (fig. 1 b), each one next to a vacant site.

Table II presents the results for isolated cobalt in a divacancy site. Figure 2b shows the electronic structure of the $\left(\mathrm{C}_{3} V \mathrm{Co} V \mathrm{C}_{3}\right)^{0}$ center. It can be described as an interaction between the anti-bonding divacancy states [33] (Fig. 2a) and the Co-related atomic orbitals (Fig. 2r). The center has a spin $\mathrm{S}=3 / 2$ and the highest occupied level has a prevailing Co $3 \mathrm{~d}$-character. In the positive charge state, the center has a $\mathrm{D}_{3 d}$ symmetry and a spin $\mathrm{S}=2$. The highest occupied level $e_{u \uparrow}^{2}$ is near the top of the VB and has a prevailing divacancy-like behavior, which resembles the result for the $\left(\mathrm{C}_{3} V \mathrm{Ni} V \mathrm{C}_{3}\right)$ complex in diamond [34]. In the negative charge state, this center has also a $\mathrm{D}_{3 d}$ symmetry and spin $\mathrm{S}=1$, with the highest occupied level being a cobalt-related orbital. In the doubly (triply) negative charge state, it has a $\mathrm{C}_{1 \mathrm{~h}}\left(\mathrm{D}_{3 d}\right)$ symmetry and spin $\mathrm{S}=1 / 2(\mathrm{~S}=0)$. Recently, the $\left(\mathrm{C}_{3} V \mathrm{Co} V \mathrm{C}_{3}\right)^{-}$center has been suggested as the microscopic model to explain the EPR data in Co-doped diamond 
[13], in the same way as the $\left(\mathrm{C}_{3} V \mathrm{Ni} V \mathrm{C}_{3}\right)^{-}$was suggested as the structure of the NE4 center [15]. However, our results indicate that the electronic structure of the $\left(\mathrm{C}_{3} V \mathrm{Co} V \mathrm{C}_{3}\right)^{-}$ center cannot be associated to a pure $3 \mathrm{~d}-t_{2}$ low spin configuration which would come from the remaining 3d-electrons of the Co impurity after all the divacancy dangling bonds were passivated, as discussed in Ref. [13].

\section{B. Cobalt-Nitrogen complexes}

We now discuss the properties of cobalt-related centers in a semi-divacancy site with several nearby substitutional nitrogen impurities. Table III presents the results for complexes involving one or two nitrogen atoms. The $\left(\mathrm{C}_{3} V \mathrm{Co} V \mathrm{C}_{2} \mathrm{~N}\right)$ center is described by one of those six neighboring carbon atoms being replaced by a nitrogen one in the $\left(\mathrm{C}_{3} V \mathrm{Co} V \mathrm{C}_{3}\right)$ precursor. The $\left(\mathrm{NC}_{2} V \mathrm{Co} V \mathrm{C}_{2} \mathrm{~N}\right)$ center is a configuration with two substitutional nitrogen atoms (in positions 3 and 6 of figure 1) at opposite sides of the divacancy, while in the $\left(\mathrm{C}_{2} \mathrm{~N} V \mathrm{Co} V \mathrm{NC}_{2}\right)$ center, the nitrogen atoms are in positions 1 and 3 . Finally, the $\left(\mathrm{C}_{3} V \mathrm{Co} V \mathrm{CN}_{2}\right)$ center has two nitrogen atoms at positions 2 and 3 . Table IV presents the results for centers involving three and four nitrogen atoms. The $\left(\mathrm{CN}_{2} V \mathrm{Co} V \mathrm{NC}_{2}\right)$ center has nitrogen atoms in positions 2,3 and 5 , while in the $\left(\mathrm{C}_{3} V \mathrm{Co} V \mathrm{~N}_{3}\right)$ center, the nitrogen atoms are in positions 2, 3 and 4 . The $\left(\mathrm{NC}_{2} V \mathrm{Co} V \mathrm{CN}_{2}\right)$ center has nitrogen atoms in positions 1 , 2 and 5. The $\left(\mathrm{CN}_{2} V \mathrm{Co} V \mathrm{~N}_{2} \mathrm{C}\right)$ center has nitrogen atoms in positions 3, 4, 5 and 6 .

The $\left(\mathrm{C}_{3} V \mathrm{Co} V \mathrm{C}_{2} \mathrm{~N}\right)$ complex, at all charge states described in table III, presents fully occupied Co 3d-related levels, such that the relevant magnetic, and probably optical, properties come from the partially occupied divacancy-related levels. An ionic model has been used to describe the electronic structure of the $\left(\mathrm{C}_{3} V \mathrm{Co} V \mathrm{C}_{2} \mathrm{~N}\right)$ centers, suggesting that the nitrogen impurity would work as a donor, giving away one electron to the $\left(\mathrm{C}_{3} V \mathrm{Co} V \mathrm{C}_{3}\right)$ precursor [13]. Although such a simple model could be invoked to explain the driving force for the complex formation [35], it is unsuitable to explain its final stable configuration. This can be understood, for example, by analyzing the electronic structure of the $\left(\mathrm{C}_{3} V \mathrm{Co} V \mathrm{C}_{2} \mathrm{~N}\right)^{0}$ complex. Considering an ionic model, this complex should have a spin $\mathrm{S}=1$, that would be obtained by taking the results of the precursor complex (Fig. 2b), and simply adding one electron to the system. Our results show otherwise, this center is diamagnetic $(\mathrm{S}=0)$, which is a result of a strong interaction among nitrogen, cobalt and carbon crystalline orbitals. 
This indicates that the covalent interactions play a fundamental role in stabilizing this complex, as it has already been shown in a number of TM-related complexes in semiconductors [36]. When a nitrogen atom replaces one of the carbon atoms, arranged in an almost octraedral environment around the Co-semi-divacancy complex (fig. 1), the symmetry lowering splits the $e_{u}$-related divacancy energy levels into two non-degenerated $a+a^{\prime}$ levels. Their covalent interaction with the $\mathrm{N}_{\mathrm{s}}$ orbitals, that introduces a non-degenerate level in the upper gap region, pushes the energy level with an $a$ representation toward the valence band, leaving the other one $\left(a^{\prime}\right)$ in the gap. This could be better understood by comparing the electronic structures of the $\left(\mathrm{C}_{3} V \mathrm{Co} V \mathrm{C}_{3}\right)^{-2}$ and $\left(\mathrm{C}_{3} V \mathrm{Co} V \mathrm{C}_{2} \mathrm{~N}\right)^{-}$complexes, that have the same number of electrons, shown respectively in figs. $3 \mathrm{a}$ and $3 \mathrm{~b}$. The nitrogen incorporation in the precursor complex alters substantially the final electronic structure of the center, i.e., the picture of a nitrogen atom just donating electrons to that precursor complex is not valid.

Table III also presents the results for Co-complexes involving two nitrogen atoms. For all configurations considered here, the centers are diamagnetic in the positive and negative charge states, while they have spin $\mathrm{S}=1 / 2$ for the neutral and doubly positive and negative charge states. Table IV presents the results for Co-complexes involving three and four nitrogen atoms. The trends on the electronic structure, as result of nitrogen incorporation, is presented in figure 3. Going from a complex with one nitrogen atom (Fig. 3b) to a complex with two nitrogen atoms (Fig. 3r), the covalent interaction is strengthened, leading to a deepening of the divacancy-related levels with a representation. The 3d-related Co levels remain almost unaffected, with respect to the valence band top, by the nitrogen incorporation. The same trend is observed for additional nitrogen incorporation, as shown in Figs. 3 d and 3e for complexes with three and four nitrogen atoms, respectively. Based on the formation energies of the Co-complexes presented in tables [II, III and IV, we find that nitrogen incorporation into the $\left(\mathrm{C}_{3} V \mathrm{Co} V \mathrm{C}_{3}\right)$ precursor is considerably favorable, suggesting that nitrogen complexing is very likely in synthetic diamond.

\section{SUMMARY}

In summary, we performed a theoretical investigation on the properties of Co-related impurity complexes in diamond. We find that the formation energy of Co in interstitial sites is considerably larger than that for Co in substitutional or divacancy sites, suggesting a 
prevailing concentration of Co in the later sites. We also show that the electronic structure of a Co impurity in substitutional or divacancy sites can be well described in terms of a vacancy model, and results from a hybridization between the vacancy-related orbitals and the Co 3d-related ones. On the other hand, the electronic structure for interstitial Co gives a low spin ground state configuration for all charge states.

These results could help into building microscopic models for the known Co-related active centers in diamond [11, 13, 14]. The center identified in Ref. [14] is fully consistent with $\mathrm{Co}_{\mathrm{i}}^{2+}$ structure in terms of symmetry $\left(\mathrm{D}_{2 \mathrm{~d}}\right)$ and electronic structure ( $\left.\operatorname{spin} \mathrm{S}=3 / 2\right)$, but the isotropic hyperfine field in the Co nucleus $(216 \mathrm{MHz})$ is smaller than the experimental hyperfine parameters observed for this center [14]. We show that the earlier proposed picture of this center, in terms of a pure $3 \mathrm{~d}^{7}$ configuration, is misleading since there is a strong covalent interaction between the impurity and its neighbors. Additionally, the large computed formation energy for interstitial TM impurities suggests that other configurations could explain this center. Although with different symmetries, we found several centers with spin $\mathrm{S}=3 / 2$, related to Co-substitutional and Co-divacancy complexes.

Some cobalt-related centers, O4, NLO2, and NWO1, have been identified with very low symmetries [11, 13], which led to interpretations that such symmetries should come from nearby defect and/or impurities, such as vacancies and nitrogen atoms, although the EPR lines could not resolve the presence of nitrogen. Our results show that cobalt centers in isolated configurations may lead to low symmetries, even without such nearby defects. Therefore, in order to discuss the low symmetry cobalt-related centers, isolated cobalt could not be ruled out. The cobalt-related $\mathrm{O} 4, \mathrm{NLO} 2$, and NWO1 centers have spin $\mathrm{S}=1 / 2$. Considering our results, they could be related to $\mathrm{Co}_{\mathrm{s}}^{0}$, but the computed isotropic hyperfine field $\left(A_{\text {iso }}\right)$ is too small to explain the experimental values.

We also found several divacancy-related Co complexes, shown in tables [II] and [V], that could explain the experimental results in terms of symmetry, spin, and hyperfine fields. The $\left(\mathrm{C}_{3} V \mathrm{Co} V \mathrm{C}_{2} \mathrm{~N}\right)^{-}$configuration was proposed to explain the $\mathrm{O} 4$ center [13]. Our results indicate that this configuration is consistent with the experimental data in terms of spin and symmetry, but the computed isotropic hyperfine field in the Co nucleus is much smaller $(22 \mathrm{MHz})$ than the experimental value measured on the $\mathrm{O} 4$ center $(197 \mathrm{MHz})$. On the other hand, the same configuration in a positive charge state, $\left(\mathrm{C}_{3} V \operatorname{Co} V \mathrm{C}_{2} \mathrm{~N}\right)^{+}$, has a higher isotropic hyperfine field in the Co nucleus $(176 \mathrm{MHz})$, being fully consistent with 
experimental data. However, there are other complexes, involving several nitrogen atoms, that are also consistent with experimental data for the $\mathrm{O} 4$ center.

For the NLO2 and NWO1 centers, our results are consistent with the proposed microscopic complexes with two nitrogen atoms, respectively $\left(\mathrm{NC}_{2} V \mathrm{Co} V \mathrm{C}_{2} \mathrm{~N}\right)$ and $\left(\mathrm{C}_{3} V \mathrm{Co} V \mathrm{CN}_{2}\right)$, but for a charge state $2+$ and not neutral, as suggested in ref. [13]. We find configurations involving two, three, and four nitrogen atoms in different charge states that would also be fully consistent with those experimental data.

Finally, we should stress that for all the centers studied here, the magnitude of the hyperfine parameters in nitrogen nuclei, $A_{i s o}\left({ }^{14} \mathrm{~N}\right)$, is inversely proportional to that in the

cobalt nuclei, $A_{\text {iso }}\left({ }^{59} \mathrm{Co}\right)$. Therefore, for those active complexes with low $A_{\text {iso }}\left({ }^{59} \mathrm{Co}\right)$, the EPR-related nitrogen hyperfine lines should be observable.

\section{Acknowledgments}

The authors acknowledge support from Brazilian agency $\mathrm{CNPq}$ and the computational facilities provided by the CENAPAD-SP.

[1] Koizumi S, Watanabe K, Hasegawa F, and Kanda H 2001 Science 2921899

[2] Balmer RS, Friel I, Woollard SM, Wort CJH, Scarsbrook GA, Coe SE, El-Hajj H, Kaiser A, Denisenko A, Kohn E, and Isberg J 2008 Philos. Trans. Royal Soc. A: Math. Phys. Eng. Sci. 366251

[3] Collins AT 2000 Diamond Relat. Mater. 9417

[4] Collins AT and Spear PM 1982 J. Phys. D - Appl. Phys. 15 L183

[5] Isoya J, Kanda H, Norris JR, Tang J, and Bowman MK 1990 Phys. Rev. B 413905

[6] Isoya J, Kanda H, and Uchida Y 1990 Phys. Rev. B 429843

[7] Pereira RN, Neves AJ, Gehlhoff W, Sobolev NA, Rino L, and Kanda H 2002 Diamond Relat. Mater. 11623

[8] Nazaré MH, Neves AJ, and Davies G 1991 Phys. Rev. B 4314196

[9] Sung CM and Tai MF 1997 Int. J. Refract. Met. Hard Mater. 15237 
[10] Lawson SC, Kanda H, Watanabe K, Kiflawi I, Sato Y, and Collins AT 1996 J. Appl. Phys. 794348

[11] Twitchen DJ, Baker JM, Newton ME, and Johnston K 2000 Phys. Rev. B 619

[12] Iakoubovskii K and Collins AT 2004 J. Phys.: Condens. Matter 166897

[13] Nadolinny VA, Baker JM, Yuryeva OP, Newton ME, Twitchen DJ, and Palyanov YN 2005 Appl. Magn. Reson. 28365

[14] Bagdasaryn VS, Markosyan EA, Matosyan MA, Torosyan OS, and Sharoyan EG 1975 Soviet Phys. Solid State 17991

[15] Nadolinny VA, Yelisseyev AP, Baker JM, Newton ME, Twitchen DJ, Lawson SC, Yuryeva OP, and Feigelson BN 1999 J. Phys.: Condens. Matter 117357

[16] Gerstmann U, Amkreutz M, and Overhof H 2000 Phys. Status Solid B 217665

[17] Goss J, Briddon PR, Jones R, and Öberg S 2004 J. Phys.: Condens. Matter 164567

[18] Larico R, Assali LVC, Machado WVM, and Justo JF 2004 Appl. Phys. Lett. 84720

[19] Larico R, Machado WVM, Assali LVC, and Justo JF 2005 Diamond Relat. Mater. 14380

[20] Johnston K, Mainwood A, Collins AT, Davies G, Twitchen D, Newton M, and Baker JM 2000 Diamond Relat. Mater. 9424

[21] Johnston K and Mainwood A 2001 Physica B 308-310 565

[22] Assali LVC, Machado WVM, Larico R, and Justo JF 2007 Diamond Relat. Mater. 16819

[23] Blaha P, Schwarz K, and Luitz J, WIEN97, A Full Potential Linearized Augmented Plane Wave Package for Calculating Crystal Properties (Karlheinz Schwarz, Techn. Universitatat Wien, Austria), 1999.

[24] Perdew JP, Burke K, and Ernzerhof M 1996 Phys. Rev. Lett. 773865

[25] Assali LVC, Machado WVM, and Justo JF 2004 Phys. Rev. B 69155212

[26] Ayres F, Assali LVC, Machado WVM, and Justo JF 2006 Appl. Phys. Lett. 88011918

[27] Assali LVC, Machado WVM, and Justo JF 2006 Appl. Phys. Lett. 89072102

[28] Watkins GD 1983 Physica B+C 117-118 9

[29] Baker JM 2003 J. Phys.: Condens. Matter. 15 S2929

[30] Baker JM, private communication.

[31] Ludwig GW and Woodbury HH 1962 Solid State Phys. 13223

[32] Dietl T, Ohno H, F. Matsukura F, Cibert J, and Ferrand D 2000 Science 2871019

[33] Assali LVC, Larico R, Machado WVM, Justo JF 2005 Mater. Sci. Forum 4831043 
[34] Larico R, Justo JF, Machado WVM, and Assali LVC 2006 Physica B 376-377 292

[35] Zhao S, Assali LVC, Justo JF, Gilmer GH, and Kimerling LC 2001 J. Appl. Phys. 902744

[36] Assali LVC and Justo JF 1998 Phys. Rev. B 583870 
TABLE I: Experimental results for Co-related EPR active centers in diamond. The table presents the symmetry, spin $(\mathrm{S})$, hyperfine parameters $\left(A_{i}, i=1,2,3\right)$, and the suggested microscopic model. The isotropic hyperfine fields $\left(A_{i s o}\right)$ were computed as an average of the measured $A_{i}$. Hyperfine parameters are given in $\mathrm{MHz}$.

\begin{tabular}{lccccccc}
\hline \hline Center & Sym. & $\mathrm{S}$ & $A_{1}$ & $A_{2}$ & $A_{3}$ & $A_{\text {iso }}$ & Model \\
\hline $\mathrm{Co}_{\mathrm{i}}^{2+(a)}$ & $\mathrm{C}_{3 v}$ or $\mathrm{D}_{2 \mathrm{~d}}$ & $3 / 2$ & 245 & 260 & 260 & 255 & $\mathrm{Co}_{\mathrm{i}}^{2+}$ \\
$\mathrm{O} 4{ }^{(b)}$ & $\mathrm{C}_{1 \mathrm{~h}}$ & $1 / 2$ & 248 & 180 & 163 & 197 & $\left(\mathrm{C}_{3} V \mathrm{Co} V \mathrm{C}_{2} \mathrm{~N}\right)^{-}$ \\
$\mathrm{NLO}{ }^{(c)}$ & $\mathrm{C}_{1 \mathrm{~h}}$ or $\mathrm{C}_{2 \mathrm{~h}}$ & $1 / 2$ & 230.8 & 183.9 & 161.2 & 192 & {$\left[\left(\mathrm{NC}_{2} V \mathrm{Co} V \mathrm{C}_{2} \mathrm{~N}\right)^{0}\right.$} \\
$\mathrm{NWO}^{(c)}$ & $\mathrm{C}_{1 \mathrm{~h} \text { or } \mathrm{C}_{2 \mathrm{~h}}} 1 / 2$ & 248 & - & 187.4 & & & $\left(\mathrm{C}_{3} V \mathrm{Co} V \mathrm{CN}_{2}\right)^{0}$ \\
\hline \hline
\end{tabular}

(a) Ref. [14]; ${ }^{(b)}$ Ref. [11]; (c) Ref. [13]. 
TABLE II: Results for isolated Co impurity centers in diamond: symmetry, spin (S), formation $\left(\mathrm{E}_{\mathrm{f}}\right)$ and transition $\left(\mathrm{E}_{\mathrm{t}}\right)$ energies, and isotropic hyperfine fields $\left(A_{\text {iso }}\right)$ at the ${ }^{59}$ Co nuclei. Energies and hyperfine fields are given in $\mathrm{eV}$ and $\mathrm{MHz}$, respectively. $\epsilon_{\mathrm{F}}$ is the Fermi energy and transition energies are given with respect to the valence band top. Theoretical approximations and numerical truncations lead to estimated errors of $\approx 0.2 \mathrm{eV}$ and $\approx 30 \mathrm{MHz}$ in the energies and hyperfine fields, respectively.

\begin{tabular}{lccccc}
\hline \multicolumn{1}{c}{ Center } & $\mathrm{Sym}$. & $\mathrm{S}$ & $\mathrm{E}_{\mathrm{f}}$ & $\mathrm{E}_{\mathrm{t}}$ & $A_{\text {iso }}$ \\
\hline $\mathrm{Co}_{\mathrm{s}}^{+}$ & $\mathrm{T}_{\mathrm{d}}$ & 0 & $3.2+\epsilon_{\mathrm{F}}$ & $3.0(+/ 0)$ & 0 \\
$\mathrm{Co}_{\mathrm{s}}^{0}$ & $\mathrm{D}_{2 \mathrm{~d}}$ & $1 / 2$ & 6.2 & & 76 \\
$\mathrm{Co}_{\mathrm{s}}^{-}$ & $\mathrm{D}_{2 \mathrm{~d}}$ & 1 & $9.8-\epsilon_{\mathrm{F}}$ & $3.6(0 /-)$ & 77 \\
$\mathrm{Co}_{\mathrm{s}}^{2-}$ & $\mathrm{T}_{\mathrm{d}}$ & $3 / 2$ & $13.8-2 \epsilon_{\mathrm{F}}$ & $4.0(-/ 2-)$ & 78 \\
$\mathrm{Co}_{\mathrm{i}}^{2+}$ & $\mathrm{D}_{2 d}$ & $3 / 2$ & $14.0+2 \epsilon_{\mathrm{F}}$ & $1.1(2+/+)$ & 216 \\
$\mathrm{Co}_{\mathrm{i}}^{+}$ & $\mathrm{T}_{\mathrm{d}}$ & 1 & $15.1+\epsilon_{\mathrm{F}}$ & $1.3(+/ 0)$ & 138 \\
$\mathrm{Co}_{\mathrm{i}}^{0}$ & $\mathrm{D}_{2 \mathrm{~d}}$ & $1 / 2$ & 16.4 & & 143 \\
$\mathrm{Co}_{\mathrm{i}}^{-}$ & $\mathrm{T}_{\mathrm{d}}$ & 0 & $18.1-\epsilon_{\mathrm{F}}$ & $1.7(0 /-)$ & 0 \\
$\left(\mathrm{C}_{3} V \mathrm{Co} V \mathrm{C}_{3}\right)^{+}$ & $\mathrm{D}_{3 \mathrm{~d}}$ & 2 & $5.5+\epsilon_{\mathrm{F}}$ & $0.3(+/ 0)$ & 110 \\
$\left(\mathrm{C}_{3} V \mathrm{Co} V \mathrm{C}_{3}\right)^{0}$ & $\mathrm{C}_{2 \mathrm{~h}}$ & $3 / 2$ & 5.8 & & 108 \\
$\left(\mathrm{C}_{3} V \mathrm{Co} V \mathrm{C}_{3}\right)^{-}$ & $\mathrm{D}_{3 \mathrm{~d}}$ & 1 & $6.5-\epsilon_{\mathrm{F}}$ & $0.7(0 /-)$ & 43 \\
$\left(\mathrm{C}_{3} V \mathrm{Co} V \mathrm{C}_{3}\right)^{2-}$ & $\mathrm{C}_{1 \mathrm{~h}}$ & $1 / 2$ & $7.4-2 \epsilon_{\mathrm{F}}$ & $0.9(-/ 2-)$ & 106 \\
$\left(\mathrm{C}_{3} V \mathrm{Co} V \mathrm{C}_{3}\right)^{3-}$ & $\mathrm{D}_{3 \mathrm{~d}}$ & 0 & $9.0-3 \epsilon_{\mathrm{F}}$ & $1.6(2-/ 3-)$ & 0 \\
\hline \hline
\end{tabular}


TABLE III: Results for Co-related defect centers in diamond involving one and two nitrogen atoms: symmetry, spin, formation and transition energies, and isotropic hyperfine fields at the ${ }^{59}$ Co nuclei.

\begin{tabular}{|c|c|c|c|c|c|}
\hline Center & Sym. & S & $\mathrm{E}_{\mathrm{f}}$ & $\mathrm{E}_{\mathrm{t}}$ & $A_{\text {iso }}$ \\
\hline$\left(\mathrm{C}_{3} V \mathrm{Co} V \mathrm{C}_{2} \mathrm{~N}\right)^{+}$ & $\mathrm{C}_{1 \mathrm{~h}}$ & $1 / 2$ & $3.9+\epsilon_{\mathrm{F}}$ & $0.8(+/ 0)$ & -176 \\
\hline$\left(\mathrm{C}_{3} V \mathrm{Co} V \mathrm{C}_{2} \mathrm{~N}\right)^{0}$ & $\mathrm{C}_{1 \mathrm{~h}}$ & 0 & 4.7 & & 0 \\
\hline$\left(\mathrm{C}_{3} V \operatorname{Co} V \mathrm{C}_{2} \mathrm{~N}\right)^{-}$ & $\mathrm{C}_{1 \mathrm{~h}}$ & $1 / 2$ & $5.7-\epsilon_{\mathrm{F}}$ & $1.0(0 /-)$ & 22 \\
\hline$\left(\mathrm{C}_{3} V \mathrm{Co} V \mathrm{C}_{2} \mathrm{~N}\right)^{2-}$ & $\mathrm{C}_{1 \mathrm{~h}}$ & 0 & $7.3-2 \epsilon_{\mathrm{F}}$ & $1.6(-/ 2-)$ & 0 \\
\hline$\left(\mathrm{NC}_{2} V \mathrm{Co} V \mathrm{C}_{2} \mathrm{~N}\right)^{2+}$ & $\mathrm{C}_{2 \mathrm{~h}}$ & $1 / 2$ & $2.4+2 \epsilon_{\mathrm{F}}$ & $0.8(2+/+)$ & 216 \\
\hline$\left(\mathrm{NC}_{2} V \mathrm{Co} V \mathrm{C}_{2} \mathrm{~N}\right)^{+}$ & $\mathrm{C}_{2 \mathrm{~h}}$ & 0 & $3.2+\epsilon_{\mathrm{F}}$ & $1.0(+/ 0)$ & 0 \\
\hline$\left(\mathrm{NC}_{2} V \operatorname{Co} V \mathrm{C}_{2} \mathrm{~N}\right)^{0}$ & $\mathrm{C}_{2 \mathrm{~h}}$ & $1 / 2$ & 4.2 & & 17 \\
\hline$\left(\mathrm{NC}_{2} V \mathrm{Co} V \mathrm{C}_{2} \mathrm{~N}\right)^{-}$ & $\mathrm{C}_{2 \mathrm{~h}}$ & 0 & $5.7-\epsilon_{\mathrm{F}}$ & $1.5(0 /-)$ & 0 \\
\hline$\left(\mathrm{NC}_{2} V \mathrm{Co} V \mathrm{C}_{2} \mathrm{~N}\right)^{2-}$ & $\mathrm{C}_{2 \mathrm{~h}}$ & $1 / 2$ & $8.9-2 \epsilon_{\mathrm{F}}$ & $3.2(-/ 2-)$ & 17 \\
\hline$\left(\mathrm{C}_{2} \mathrm{~N} V \mathrm{Co} V \mathrm{NC}_{2}\right)^{2+}$ & $\mathrm{C}_{2}$ & $1 / 2$ & $2.5+2 \epsilon_{\mathrm{F}}$ & $0.5(2+/+)$ & -251 \\
\hline$\left(\mathrm{C}_{2} \mathrm{~N} V \mathrm{Co} V \mathrm{NC}_{2}\right)^{+}$ & $\mathrm{C}_{2}$ & 0 & $3.0+\epsilon_{\mathrm{F}}$ & $0.9(+/ 0)$ & 0 \\
\hline$\left(\mathrm{C}_{2} \mathrm{~N} V \mathrm{Co} V \mathrm{NC}_{2}\right)^{0}$ & $\mathrm{C}_{2}$ & $1 / 2$ & 3.9 & & 139 \\
\hline$\left(\mathrm{C}_{2} \mathrm{~N} V \mathrm{Co} V \mathrm{NC}_{2}\right)^{-}$ & $\mathrm{C}_{2}$ & 0 & $5.2-\epsilon_{\mathrm{F}}$ & $1.3(0 /-)$ & 0 \\
\hline$\left(\mathrm{C}_{2} \mathrm{~N} V \mathrm{Co} V \mathrm{NC}_{2}\right)^{2-}$ & $\mathrm{C}_{2}$ & $1 / 2$ & $9.2-2 \epsilon_{\mathrm{F}}$ & $4.0(-/ 2-)$ & 8 \\
\hline$\left(\mathrm{C}_{3} V \mathrm{Co} V \mathrm{CN}_{2}\right)^{2+}$ & $\mathrm{C}_{1 \mathrm{~h}}$ & $1 / 2$ & $2.2+2 \epsilon_{\mathrm{F}}$ & $0.5(2+/+)$ & 153 \\
\hline$\left(\mathrm{C}_{3} V \mathrm{Co} V \mathrm{CN}_{2}\right)^{+}$ & $\mathrm{C}_{1 \mathrm{~h}}$ & 0 & $2.7+\epsilon_{\mathrm{F}}$ & $0.9(+/ 0)$ & 0 \\
\hline$\left(\mathrm{C}_{3} V \mathrm{Co} V \mathrm{CN}_{2}\right)^{0}$ & $\mathrm{C}_{1 \mathrm{~h}}$ & $1 / 2$ & 3.6 & & -95 \\
\hline$\left(\mathrm{C}_{3} V \mathrm{Co} V \mathrm{CN}_{2}\right)^{-}$ & $\mathrm{C}_{1 \mathrm{~h}}$ & 0 & $5.0-\epsilon_{\mathrm{F}}$ & $1.4(0 /-)$ & 0 \\
\hline$\left(\mathrm{C}_{3} V \mathrm{Co} V \mathrm{CN}_{2}\right)^{2-}$ & $\mathrm{C}_{1 \mathrm{~h}}$ & $1 / 2$ & $8.8-2 \epsilon_{\mathrm{F}}$ & $3.8(-/ 2-)$ & 6 \\
\hline
\end{tabular}


TABLE IV: Results for Co-related defect centers in diamond involving three and four nitrogen atoms: symmetry, spin, formation and transition energies, and isotropic hyperfine fields at the ${ }^{59}$ Co nuclei.

\begin{tabular}{lccccc}
\hline \hline Center & $\mathrm{Sym}$. & $\mathrm{S}$ & $\mathrm{E}_{\mathrm{f}}$ & $\mathrm{E}_{\mathrm{t}}$ & $A_{\text {iso }}$ \\
\hline$\left(\mathrm{CN}_{2} V \mathrm{Co} V \mathrm{NC}_{2}\right)^{+}$ & $\mathrm{C}_{1 \mathrm{~h}}$ & $1 / 2$ & $1.9+\epsilon_{\mathrm{F}}$ & $0.9(+/ 0)$ & 274 \\
$\left(\mathrm{CN}_{2} V \mathrm{Co} V \mathrm{NC}_{2}\right)^{0}$ & $\mathrm{C}_{1 \mathrm{~h}}$ & 0 & 2.8 & & 0 \\
$\left(\mathrm{CN}_{2} V \mathrm{Co} V \mathrm{NC}_{2}\right)^{-}$ & $\mathrm{C}_{1 \mathrm{~h}}$ & $1 / 2$ & $6.8-\epsilon_{\mathrm{F}}$ & $4.0(0 /-)$ & 9 \\
$\left(\mathrm{C}_{3} V \mathrm{Co} V \mathrm{~N}_{3}\right)^{+}$ & $\mathrm{C}_{1 \mathrm{~h}}$ & $1 / 2$ & $1.4+\epsilon_{\mathrm{F}}$ & $1.0(+/ 0)$ & 257 \\
$\left(\mathrm{C}_{3} V \mathrm{Co} V \mathrm{~N}_{3}\right)^{0}$ & $\mathrm{C}_{3 v}$ & 0 & 2.4 & & 0 \\
$\left(\mathrm{C}_{3} V \mathrm{Co} V \mathrm{~N}_{3}\right)^{-}$ & $\mathrm{C}_{3 v}$ & $1 / 2$ & $6.4-\epsilon_{\mathrm{F}}$ & $4.0(0 /-)$ & 9 \\
$\left(\mathrm{NC}_{2} V \mathrm{Co} V \mathrm{CN}_{2}\right)^{2+}$ & $\mathrm{C}_{1}$ & 0 & $1.0+2 \epsilon_{\mathrm{F}}$ & $0.9(2+/+)$ & 0 \\
$\left(\mathrm{NC}_{2} V \mathrm{Co} V \mathrm{CN}_{2}\right)^{+}$ & $\mathrm{C}_{1}$ & $1 / 2$ & $1.9+\epsilon_{\mathrm{F}}$ & $1.3(+/ 0)$ & 29 \\
$\left(\mathrm{NC}_{2} V \mathrm{Co} V \mathrm{CN}_{2}\right)^{0}$ & $\mathrm{C}_{1}$ & 0 & 3.2 & & 0 \\
$\left(\mathrm{NC}_{2} V \mathrm{Co} V \mathrm{CN}_{2}\right)^{-}$ & $\mathrm{C}_{1}$ & $1 / 2$ & $7.4-\epsilon_{\mathrm{F}}$ & $4.2(0 /-)$ & 82 \\
$\left(\mathrm{CN}_{2} V \mathrm{Co} V \mathrm{~N}_{2} \mathrm{C}\right)^{2+}$ & $\mathrm{C}_{2 \mathrm{~h}}$ & $1 / 2$ & $0.1+2 \epsilon_{\mathrm{F}}$ & $1.0(2+/+)$ & 13 \\
$\left(\mathrm{CN}_{2} V \mathrm{Co} V \mathrm{~N}_{2} \mathrm{C}\right)^{+}$ & $\mathrm{C}_{2 \mathrm{~h}}$ & 0 & $1.1+\epsilon_{\mathrm{F}}$ & $3.6(+/ 0)$ & 0 \\
$\left(\mathrm{CN}_{2} V \mathrm{Co} V \mathrm{~N}_{2} \mathrm{C}\right)^{0}$ & $\mathrm{C}_{2 \mathrm{~h}}$ & $1 / 2$ & 4.7 & & 210 \\
$\left(\mathrm{CN}_{2} V \mathrm{Co} V \mathrm{~N}_{2} \mathrm{C}\right)^{-}$ & $\mathrm{C}_{2 \mathrm{~h}}$ & 1 & $8.9-\epsilon_{\mathrm{F}}$ & $4.2(0 /-)$ & 202 \\
\hline \hline
\end{tabular}


FIG. 1: (a) Microscopic configuration of a Co impurity between two carbon vacancies $\left(\mathrm{C}_{3} V \mathrm{Co} V \mathrm{C}_{3}\right)$. The six nearest neighboring carbon atoms are located in the edges of the gray triangles. One set $(1,5$, and 6 atoms $)$ is next to one of the vacant sites while the other $(2,3$, and 4 atoms) is next to the other vacant site. (b) Schematic representation of the configuration in a (111) plane.

FIG. 2: The calculated electronic structure of the $\left(\mathrm{C}_{3} V \text { Co } V \mathrm{C}_{3}\right)^{0}$ center (b). This structure comes from an interaction between the neutral divacancy levels (in $\mathrm{D}_{3 d}$ symmetry) (a) and the Co atomic states $\left(\mathrm{d}=2 e_{g}+a_{1 g}\right)(\mathrm{c})$. Levels with spin up (down) are represented by $\uparrow(\downarrow)$ arrows. The number of filled (open) circles represent the electronic (hole) occupation of each level.

FIG. 3: Electronic structure of the Co-related centers: (a) $\left(\mathrm{C}_{3} V \mathrm{Co} V \mathrm{C}_{3}\right)^{2-}$, (b) $\left(\mathrm{C}_{3} V \mathrm{Co} V \mathrm{C}_{2} \mathrm{~N}\right)^{-}$, (c) $\left(\mathrm{NC}_{2} V \mathrm{Co} V \mathrm{C}_{2} \mathrm{~N}\right)^{0}$, (d) $\left(\mathrm{NC}_{2} V \mathrm{Co} V \mathrm{CN}_{2}\right)^{+}$, (e) $\left(\mathrm{CN}_{2} V \mathrm{Co} V \mathrm{~N}_{2} \mathrm{C}\right)^{2+}$, all with spin $\mathrm{S}=1 / 2$. Levels with spin up (down) are represented by $\uparrow(\downarrow)$ arrows. The number of filled (open) circles represent the electronic (hole) occupation of each level. 
(a)

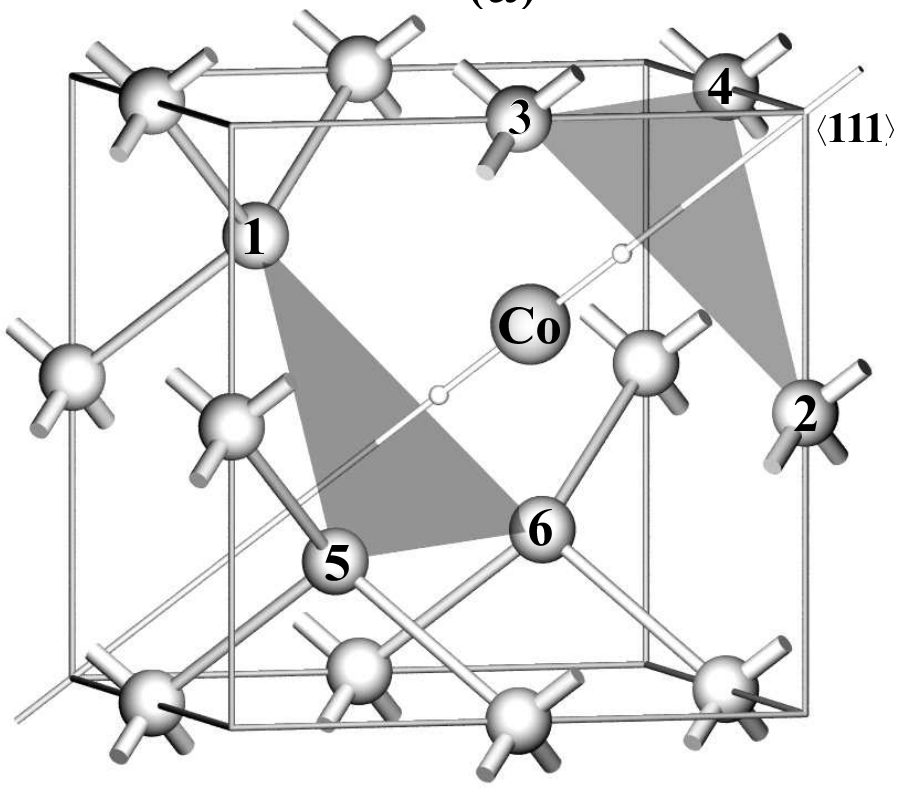

(b)

(1)

(3)

(5)

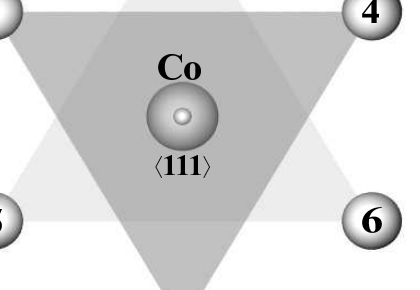

2 


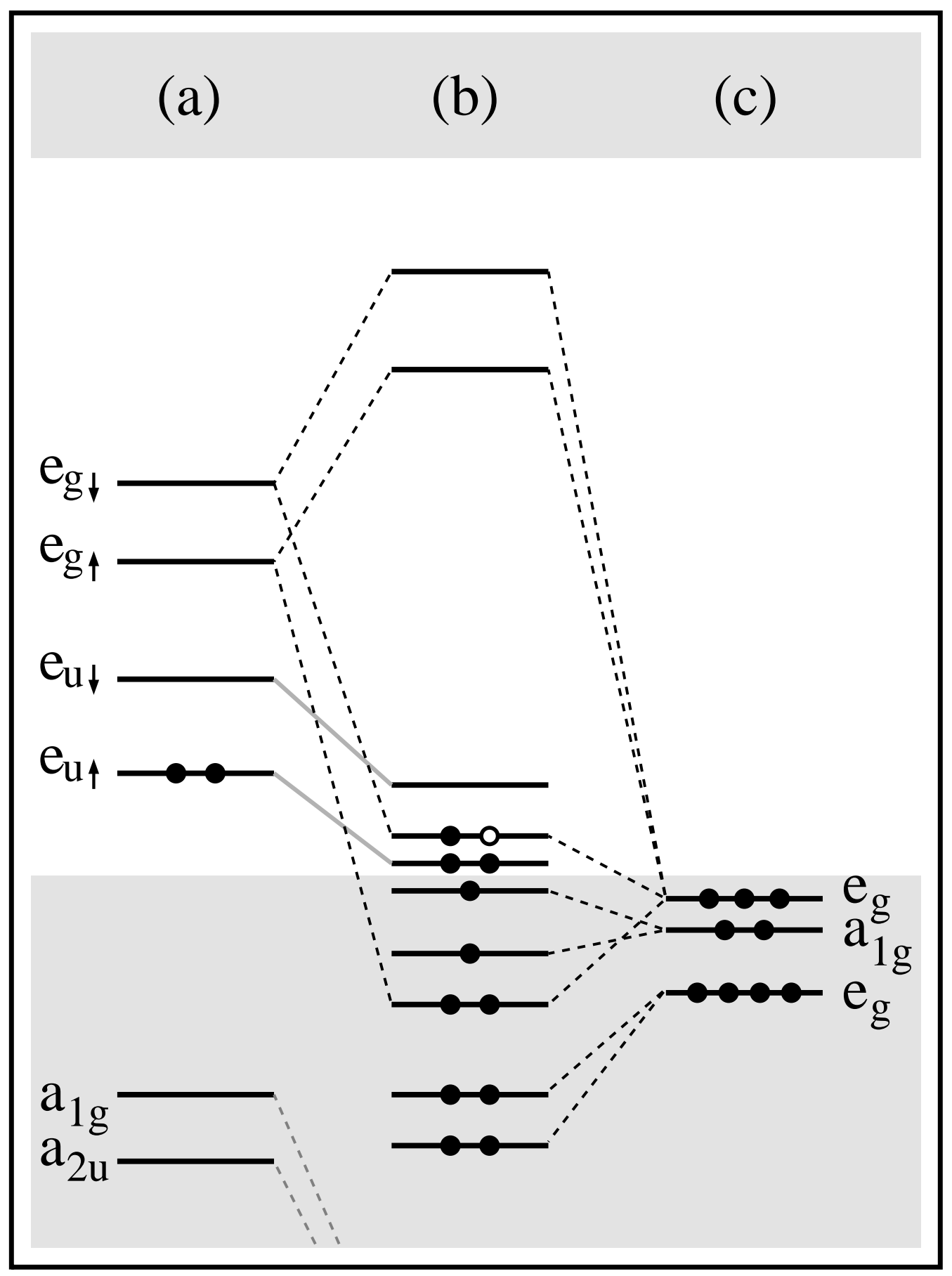




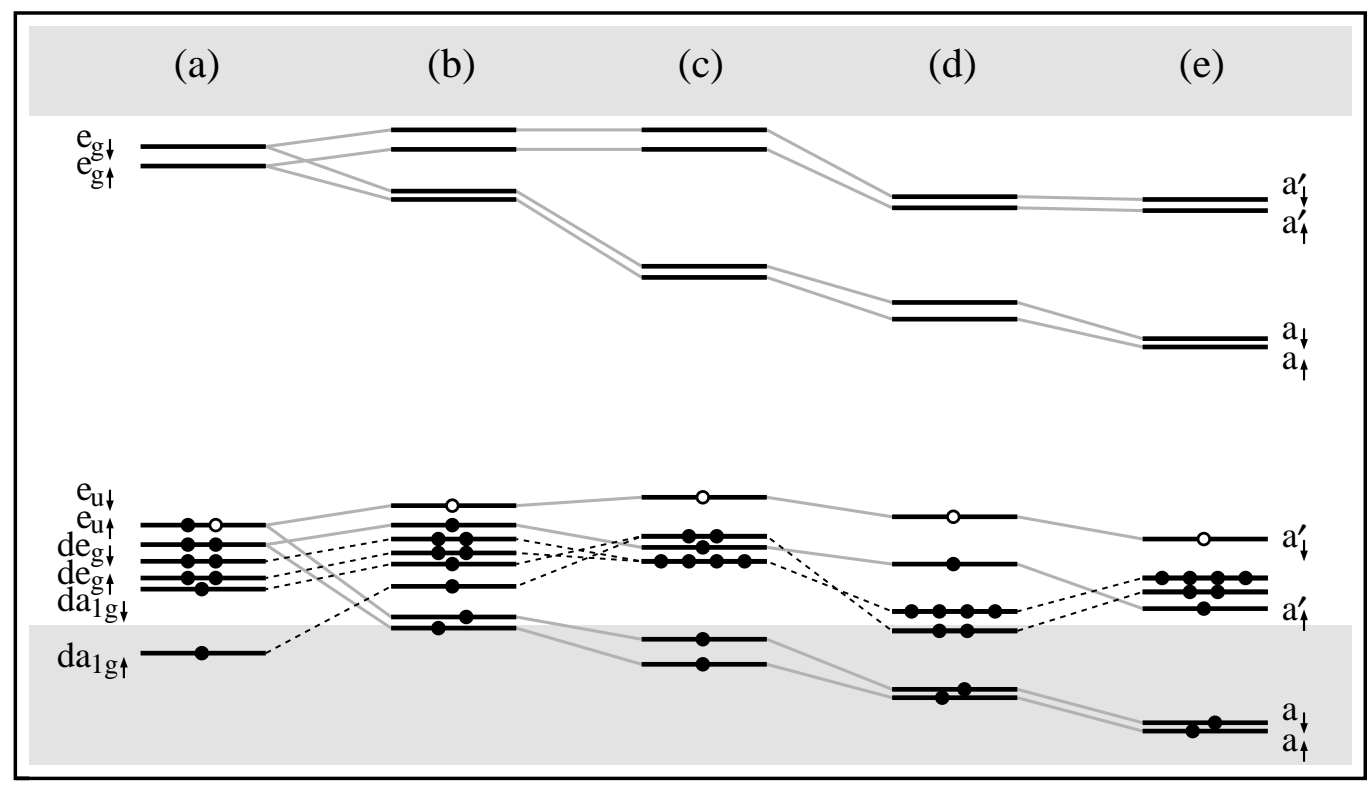

